\title{
Value distribution of higher order differential-difference polynomial of an entire function
}

\author{
Renukadevi Dyavanal and Jyoti Muttagi \\ Department of Mathematics, Karnatak University, Dharwad, India
}

Received: 15 June 2018, Accepted: 22 January 2019

Published online: 17 March 2019.

\begin{abstract}
This paper deals with value distribution of higher order differential-difference operators of an entire function and obtained results improve some classical results on differential polynomials and deduce results of K. Liu, T.B. Cao and X.L. Liu [17] as particular
\end{abstract} case of our results.

Keywords: Nevanlinna theory, differential-difference polynomial of a function, difference operator etc.

\section{Introduction and main results}

We adopt fundamental results and the standard notations of the Neavanlinna theory of meromorphic functions as explained in ([7], [11] and [20]). A meromorphic function $f$ means meromorphic in the whole complex plane. If no poles occur, then $f$ reduces to an entire function. Given a meromorphic function $f(z)$, recall that $\alpha(z) \not \equiv 0, \infty$ is a small function with respect to $f(z)$ if $T(r, \alpha)=S(r, f)$, where $S(r, f)$ is used to denote any quantity satisfying $S(r, f)=o(T(r, f))$ and $r \rightarrow \infty$ outside of a possible exceptional set of finite logarithmic measure. The order $\rho(f)$ is defined by

$$
\rho(f)=\limsup _{r \rightarrow \infty} \frac{\log ^{+} T(r, f)}{\log r}
$$

A polynomial $p(z)$ is called a Borel exceptional polynomial of $f(z)$ whenever

$$
\lambda(f(z)-p(z))=\limsup _{r \rightarrow \infty} \frac{\log ^{+} N\left(r, \frac{1}{f(z)-p(z)}\right)}{\log r}<\rho(f),
$$

where $\lambda(f(z)-p(z))$ is the exponent of convergence of zeros of $f(z)-p(z)$. In this paper, we assume that $c$ is a nonzero complex constant, $n$ is a positive integer and $\mathrm{k}$ is a nonnegative integer, unless otherwise specified.

Recently the topic of distribution of values of differential polynomials of different types in the complex plane $\mathbb{C}$ has attracted many mathematicians, a number of papers have focused on the zeros of $f(z)$ and its derivatives can be found in ([10],[11] and [21]).

In 2017, K. Liu, T.B. Cao and X.L. Liu [17] investigated some classical results on the distribution of zeros for differential polynomials and differential-difference polynomials and obtained the following results. 
Theorem 1. Let $f(z)$ be a transcendental entire function of finite order. If $n \geq 1, k \geq 0$ and

$$
N\left(r, \frac{1}{f}\right)=S(r, f),
$$

then $\left[f(z)^{n} f(z+c)\right]^{(k)}-\alpha(z)$ has infinitely many zeros, where $\alpha(z)$ is a nonzero small function with respect to $f(z)$.

Theorem 2. Let $f(z)$ be a transcendental entire function of finite order, which is not a periodic function with period c. If $n \geq 1, k \geq 0$ and $N\left(r, \frac{1}{f}\right)=S(r, f)$, then $\left[f(z)^{n} \Delta_{c} f\right]^{(k)}-\alpha(z)$ has infinitely many zeros.

Theorem 3. Let $f(z)$ be a transcendental entire function of finite order. If $n \geq \frac{k}{2}+1, k \geq 0$ and $f$ has infinitely many multiorder zeros, then $\left[f(z)^{n} f(z+c)\right]^{(k)}-p(z)$, has infinitely many zeros, where $p(z)$ is a nonzero polynomial.

Theorem 4. Let $f(z)$ be a transcendental entire function of finite order, which is not a periodic function with period $c$. If $n \geq \frac{k}{2}+1, k \geq 0$ and $f$ has infinitely many multiorder zeros, then $\left[f(z)^{n}(f(z+c)-f(z))\right]^{(k)}-p(z)$, has infinitely many zeros.

Theorem 5. Let $f(z)$ be a transcendental entire function of finite order, let $p(z)$ be a nonzero polynomial, and let $n \geq 1$, $k \geq 0$. If $f$ has a Borel exceptional polynomial $q(z)$, then $\left[f(z)^{n} f(z+c)\right]^{(k)}-p(z)$ has infinitely many zeros except $f(z)=$ $q(z)+A q(z) e^{\alpha z}, n=1$, and $p(z)=[q(z) q(z+c)]^{(k)}$, where $e^{\alpha c}=-1$ and $A$ is a nonzero constant.

Theorem 6. Let $f(z)$ be a transcendental entire function of finite order, which is not a periodic function with period $c$ and let $n \geq 1, k \geq 0$. If $f$ has a Borel exceptional polynomial $q(z)$, then $\left[f(z)^{n}(f(z+c)-f(z))\right]^{(k)}-p(z)$ has infinitely many zeros, except the cases $f(z)=q(z)+h e^{\alpha z}, n=1$, and $p(z)=[q(z)(q(z+c)-q(z))]^{(k)}$, where $e^{\alpha c}=1$.

In this paper, above theorems are generalized for higher order differential-difference operators as follows.

Theorem 7. Let $f(z)$ be a transcendental entire function of finite order, which is not a periodic function with period $c$. If $n$ and $m$ are positive integers with $n \geq 1, k \geq 0$ and $N\left(r, \frac{1}{f}\right)=S(r, f)$, then $\left[f(z)^{n} \Delta_{c}^{m} f\right]^{(k)}-\alpha(z)$ has infinitely many zeros, where $\alpha(z)$ is a nonzero small function with respect to $f(z)$.

Remark. If $m=1$ in Theorem 7, then Theorem 7 reduces to Theorem 2.

Theorem 8. Let $f(z)$ be a transcendental entire function of finite order, which is not a periodic function with period c. If $n$ and $m$ are positive integers with $n \geq \frac{k}{2}+1, k \geq 0$ and f has infinitely many multiorder zeros, then $\left[f(z)^{n} \Delta_{c}^{m} f\right]^{(k)}-p(z)$, has infinitely many zeros, where $p(z)$ is a nonzero polynomial.

Remark. If $m=1$ in Theorem 8 , then Theorem 8 reduces to Theorem 4 .

Theorem 9. Let $f(z)$ be a transcendental entire function of finite order, which is not a periodic function with period $c$ and If $n$ and $m$ are positive integers with $n \geq 1, k \geq 0$. If f has a Borel exceptional polynomial $q(z)$, then $\left[f(z)^{n} \Delta_{c}^{m} f\right]^{(k)}-p(z)$ has infinitely many zeros, except the cases $f(z)=q(z)+h(z) e^{\alpha z}, n=1$, and $p(z)=[q(z)(q(z+c)-q(z))]^{(k)}$, where $e^{\alpha c}=1, \alpha$ is a nonzero constant and $h(z)$ is a nonzero entire function with $\rho(h)<\rho(f)$.

Remark. If $m=1$ in Theorem 9, then Theorem 9, reduces to Theorem 6. 


\section{Some lemmas}

We need the following Lemmas to prove our results.

Lemma 1. Let $f$ be a transcendental meromorphic function of finite order. Then

$$
m\left(r, \frac{f(z+c)}{f(z)}\right)=S(r, f)
$$

Lemma 2. [6] Let $f(z)$ be a transcendental meromorphic function of finite order. Then

$$
T(r, f(z+c))=T(r, f)+S(r, f) .
$$

Lemma 3. [20] Let $f(z)$ be a transcendental meromorphic function and let $n$ be a positive integer. Then

$$
T\left(r, f^{(n)}\right) \leq T(r, f)+n \bar{N}(r, f)+S(r, f)
$$

Lemma 4. Let $f(z)$ be a transcendental meromorphic function of finite order with $N(r, f)+N\left(r, \frac{1}{f}\right)=S(r, f)$ and $F(z)=$ $f(z)^{n} \Delta_{c}^{m} f$, where $n$ and $m$ are positive integer. Then

$$
(n+1) T(r, f)+S(r, f) \leq T(r, F)
$$

Proof. From the first fundamental theorem, Lemma 1 and the assumption, we obtain

$$
(n+1) T(r, f(z))=T\left(r, f(z)^{n+1}\right)=T\left(r, \frac{f(z) F(z)}{\Delta_{c}^{m} f}\right) \leq T(r, F(z))+T\left(r, \frac{f(z)}{\Delta_{c}^{m} f}\right)+S(r, f) \leq T(r, F(z))+S(r, f) .
$$

Lemma 5. [2] Let $g(z)$ be a transcendental meromorphic function of order $\sigma(f)<1, h>0$. Then there exists an $\varepsilon$-set $E$ such that

$$
\frac{g^{\prime}(z+c)}{g(z+c)} \rightarrow 0
$$

and

$$
\frac{g(z+c)}{g(z)} \rightarrow 1
$$

as $z \rightarrow \infty$ in $\mathbf{C} \backslash E$, uniformly in c for $|c| \leq h$. Further, $E$ can be chosen so that, for large $z \notin E$, the function $g$ has no zeros or poles in $|\zeta-z| \leq h$.

Lemma 6. [21], Lemma 1 Let $f$ be a nonconstant meromorphic function and let $\alpha(z)$ be a small function of $f$ such that $\alpha(z) \neq 0, \infty$. Then

$$
T(r, f) \leq \bar{N}(r, f)+N\left(r, \frac{1}{f}\right)+N\left(r, \frac{1}{f^{(k)}-\alpha}\right)-N\left(r, \frac{1}{\left(\frac{f^{(k)}}{\alpha}\right)}\right)+S(r, f)
$$

Lemma 7. [20], Theorem 1.62 Let $f_{j}(z)$ be a meromorphic functions and let $f_{k}(z), k=1,2, \ldots, n-1$, be not constant satisfying the relation

$$
\sum_{j=1}^{n} f_{j}=1
$$


with $n \geq 3$. If $f_{n}(z) \not \equiv 0$ and

$$
\sum_{j=1}^{n} N\left(r, \frac{1}{f_{j}}\right)+(n-1) \sum_{j=1}^{n} \bar{N}\left(r, f_{j}\right)<(\lambda+o(1)) T\left(r, f_{k}\right)
$$

where $\lambda<1, k=1,2, \ldots, n-1$, then $f_{n}(z) \equiv 1$.

Lemma 8. [20], Theorem 1.51 Let $f_{j}(z), j=1,2, \ldots, n, n \geq 2$, be meromorphic functions and let $g_{j}(z) j=1,2, \ldots, n$, be entire functions satisfying:

(1) $\sum_{j=1}^{n} f_{j}(z) e^{g_{j}(z)} \equiv 0$,

(2) $g_{j}(z)-g_{k}(z)$ is not constant for $1 \leq j<k \leq n$,

(3) for $1 \leq j \leq n, 1 \leq h<k \leq n, T\left(r, f_{j}\right)=o\left(T\left(r, e^{g_{h}-g_{k}}\right)\right)(r \rightarrow \infty, r \notin E)$, where $E \subset(1, \infty)$ is of finite linear measure or finite logarithmic measure.

Then $f_{j}(z) \equiv 0, j=1,2, \ldots, n$.

Lemma 9. [6], Theorem 9.2 Let $A_{0}(z), \ldots, A_{n}(z)$ be entire functions for which there exists an integer $l, 0 \leq l \leq n$, such that

$$
\rho\left(A_{l}(z)\right)>\max _{0 \leq l \leq n, j \neq l} \rho\left(A_{j}(z)\right)
$$

If $f(z)$ is a meromorphic solution of

$$
A_{n}(z) y\left(z+c_{n}\right)+\ldots+A_{1}(z) y\left(z+c_{1}\right)+A_{0}(z) y(z)=0
$$

then

$$
\rho(f) \geq \rho\left(A_{l}(z)\right)+1
$$

Lemma 10. [5], Theorem 1.2 Let $P_{0}(z), \ldots, P_{n}(z)$ be polynomials such that $P_{n}(z) P_{0}(z) \not \equiv 0$ satisfying the relation

$$
\operatorname{deg}\left(P_{n}(z)+\ldots+P_{0}(z)\right)=\max \left\{\operatorname{deg} P_{j}(z): j=0, \ldots, n\right\} \geq 1 .
$$

Then every finite order meromorphic solution $f(z)(\not \equiv 0)$ of

$$
P_{n}(z) f\left(z+c_{n}\right)+\ldots+P_{1}(z) f\left(z+c_{1}\right)+P_{0}(z) f(z)=0
$$

satisfies the inequality $\rho(f) \geq 1$.

Proof. (Proof of theorem 7) Let $F(z)=f(z)^{n} \Delta_{c}^{m} f$, that is $F(z)=f(z)^{n} \Delta_{c}^{m-1}\left(\Delta_{c} f\right)$. Since by hypothesis

$$
N(r, f)+N\left(r, \frac{1}{f}\right)=S(r, f) .
$$

We conclude that

$$
\begin{aligned}
N(r, F)+N\left(r, \frac{1}{F}\right) & \leq N\left(r, \frac{1}{f(z)^{n} \Delta_{c}^{m} f}\right) \leq N\left(r, \frac{1}{f(z)^{n}}\right)+N\left(r, \frac{1}{\Delta_{c}^{m} f}\right) \leq T\left(r, \Delta_{c}^{m} f\right)+S(r, f) \\
& \leq m\left(r, \Delta_{c}^{m} f\right)+N\left(r, \Delta_{c}^{m} f\right)+S(r, f) \leq m\left(r, \frac{\Delta_{c}^{m} f}{f}\right)+m(r, f)+S(r, f) \leq T(r, f)+S(r, f) .
\end{aligned}
$$


We have

$$
\begin{aligned}
T(r, F) & =T\left(r, f(z)^{n} \Delta_{c}^{m} f\right) \leq T\left(r, f(z)^{n}\right)+T\left(r, \Delta_{c}^{m} f\right)+S(r, f) \leq n T(r, f)+m\left(r, \Delta_{c}^{m} f\right)+N\left(r, \Delta_{c}^{m} f\right)+S(r, f) \\
& \leq n T(r, f)+m\left(r, \frac{\Delta_{c}^{m} f}{f}\right)+m(r, f)+S(r, f) \leq n T(r, f)+m(r, f)+S(r, f) \\
& \leq n T(r, f)+T(r, f)+S(r, f) \leq(n+1) T(r, f)+S(r, f) .
\end{aligned}
$$

From the above inequality and Lemma 4, we get

$$
(n+1) T(r, f)+S(r, f)=T(r, F) .
$$

Using, this with Lemma 6 and (1)

$$
\begin{aligned}
(n+1) T(r, f)+S(r, f) & \leq \bar{N}(r, F)+N\left(r, \frac{1}{F}\right)+N\left(r, \frac{1}{F^{(k)}-\alpha}\right)+S(r, F) \\
& \leq N(r, F)+N\left(r, \frac{1}{F}\right)+N\left(r, \frac{1}{F^{(k)}-\alpha}\right)+S(r, F) \\
& \leq T(r, f)+N\left(r, \frac{1}{F^{(k)}-\alpha}\right)+S(r, f) \\
n T(r, f) & \leq N\left(r, \frac{1}{F^{(k)}-\alpha}\right)+S(r, f) .
\end{aligned}
$$

Since $n \geq 1$ we conclude that $\left[f(z)^{n} \Delta_{c}^{m} f\right]^{(k)}-\alpha(z)$ has infinitely many zeros.

Proof. (Proof of theorem 8) Let

$$
F(z)=f(z)^{n} \Delta_{c}^{m} f .
$$

Assume that $F^{(k)}(z)-p(z)$ has finitely many zeros. From Hadamard factorization theorem, we have

$$
F^{(k)}(z)-p(z)=h(z) e^{q(z)},
$$

where $h(z)$ is a nonzero polynomial and $q(z)$ is a nonconstant polynomial, otherwise if $q(z)=A$, where $A$ is a constant, then $F^{(k)}(z)-p(z)=h(z) e^{A}$. This implies that $F(z)=f(z)^{n} \Delta_{c}^{m} f$ is also a polynomial, which contradicts $f(z)$ is transcendental entire function. Differentiating (2), we get

$$
F^{(k+1)}(z)-p^{\prime}(z)=\left[h^{\prime}(z)+h(z) q^{\prime}(z)\right] e^{q(z)}
$$

Combining (2) with (3) and eliminating $e^{q(z)}$, we obtain

$$
\frac{F^{(k+1)}(z)}{F^{(k)}(z)}=\frac{h^{\prime}(z)+h(z) q^{\prime}(z)}{h(z)}+\left[p^{\prime}(z)-\frac{h^{\prime}(z)+h(z) q^{\prime}(z)}{h(z)} p(z)\right] \frac{1}{F^{(k)}(z)} .
$$

We note that poles of $\frac{F^{(k+1)}(z)}{F^{(k)}(z)}$ on the left hand side of (4) must be simple. If $f$ has infinitely many multiorder zeros and

$$
n \geq \frac{k}{2}+1
$$


then we can find $z_{0}$ which is a zero of $f$ and not a zero of $h(z)$ and $p^{\prime}(z)-\frac{h^{\prime}(z)+h(z) q^{\prime}(z)}{h(z)} p(z)$. Thus, the poles of right hand side of (4) must be multiorder, a contradiction.

Proof. ( Proof of theorem 9) Assume that $\rho(f)=s$, where $s$ is a positive integer. Then the transcendental entire function $f(z)$ can be represented as

$$
f(z)=q(z)+h(z) e^{\alpha z^{s}}
$$

where $\alpha$ is a nonzero constant and $h(z)$ is a nonzero entire function with

$$
\lambda(h) \leq \rho(h)<\rho(f)=s .
$$

It follows from (5) that

$$
f(z+c)=q(z+c)+h(z+c) e^{\alpha(z+c)^{s}}=q(z+c)+h_{1}(z) e^{\alpha z^{s}}
$$

where

$$
h_{1}(z)=h(z+c) e^{\alpha\left(C_{s}^{1} z^{s-1} c+C_{s}^{2} z^{s-2} c^{2}+\ldots+C_{s}^{s-1} z c^{s-1}+c^{s}\right)} .
$$

Thus

$$
f(z+c)-f(z)=q(z+c)-q(z)+\left(h_{1}(z)-h(z)\right) e^{\alpha z^{s}}=q_{1}(z)+h_{2}(z) e^{\alpha z^{s}}
$$

We have

$$
\Delta_{c}^{m} f=\Delta_{c}^{m-1}(f(z+c)-f(z))=\Delta_{c}^{m-1}\left[q(z+c)-q(z)+\left(h_{1}(z)-h(z)\right) e^{\alpha z^{s}}\right]=\Delta_{c}^{m-1}\left[q_{1}(z)+h_{2}(z) e^{\alpha z^{s}}\right]
$$

where $q_{1}(z)=q(z+c)-q(z)$ and $h_{2}(z)=h_{1}(z)-h(z)$. Suppose that $\left[f(z)^{n} \Delta_{c}^{m} f\right]^{(k)}-p(z)$ has finitely many zeros. Thus, from the Hadamard factorization theorem, we obtain

$$
\left[f(z)^{n} \Delta_{c}^{m} f\right]^{(k)}-p(z)=C(z) e^{\gamma z^{s}} .
$$

This implies

$$
\left[f(z)^{n} \Delta_{c}^{m-1}(f(z+c)-f(z))\right]^{(k)}-p(z)=C(z) e^{\gamma z^{s}}
$$

where $C(z)$ is an entire function with finitely many zeros of order $\rho(C)<s$ and $\gamma$ is a nonzero constant.

Case 1 . Let $k=0$ and $n=1$ in (8), we get

$$
\left[f(z) \Delta_{c}^{m-1}(f(z+c)-f(z))\right]-p(z)=C(z) e^{\gamma z^{s}} .
$$

Substituting $f(z)=q(z)+h(z) e^{\alpha z^{s}}$ in (9) we get

$$
\left[q(z)+h(z) e^{\alpha z^{s}}\right]\left[\Delta_{c}^{m-1}\left(q(z+c)+h(z+c) e^{\alpha(z+c)^{s}}-q(z)-h(z) e^{\alpha z^{s}}\right)\right]-p(z)=C(z) e^{\gamma z^{s}}
$$

We get

$$
\begin{gathered}
h(z)\left[\Delta_{c}^{m-1}\left(h_{1}(z)-h(z)\right)\right] e^{2 \alpha z^{s}}+\left[h(z)\left(\Delta_{c}^{m-1}(q(z+c)-q(z))\right)+q(z)\left(\Delta_{c}^{m-1}\left(h_{1}(z)-h(z)\right)\right)\right] e^{\alpha z^{s}} \\
=p(z)-q(z)\left[\Delta_{c}^{m-1}(q(z+c)-q(z))\right]+C(z) e^{\gamma z^{s}} .
\end{gathered}
$$

Subcase 1.1. If $p(z)-q(z)\left[\Delta_{c}^{m-1}(q(z+c)-q(z))\right] \equiv 0$. If $h_{1}(z)=h(z)$, then $\alpha=\gamma$, follows from the equation presented above. It follows from (10) and Lemma 9 that $s=1$. Therefore $h(z)=h(z+c) e^{\alpha c}$. By using Lemma 5 we conclude that 
$h(z)$ is a constant and $e^{\alpha c}=1$. Thus, $f(z)=q(z)+h e^{\alpha z}$, where $e^{\alpha c}=1$.

If $h_{1}(z) \quad \neq \equiv h(z)$, then $\gamma=2 \alpha$. From Lemma 8 , we obtain $\left[h(z)\left(\Delta_{c}^{m-1}(q(z+c)-q(z))\right)+q(z)\left(\Delta_{c}^{m-1}\left(h(z+c) e^{\alpha}\left(C_{s}^{1} z^{s-1} c+\ldots+C_{s}^{s-1} z c^{s-1}+c^{s}\right)-h(z)\right)\right)\right] e^{\alpha z^{s}}=0$. By Lemma 9, we get $s=1$. therefore,

$$
\left[h(z)\left(\Delta_{c}^{m-1}(q(z+c)-q(z))\right)+q(z)\left(\Delta_{c}^{m-1}\left(h(z+c) e^{\alpha c}-h(z)\right)\right)\right]=0 .
$$

Since $\rho(h)<1$ in Lemma 10, we see that either $q(z)$ must be constant or $e^{\alpha c}=1$ if $q(z)$ is not a constant. If $\mathrm{q}(\mathrm{z})$ is a constant from Lemma 5 then $h(z)$ reduces a constant. Hence, $f(z)$ is a periodic function with period $c$, a contradiction.

If $e^{\alpha c}=1$ and $q(z)$ is not a constant, in view of (10), we get $h(z)\left[\Delta_{c}^{m-1}(h(z+c)-h(z))\right]=C(z)$. Combining this with the inequality $\rho(h)<1$ and the fact that $h(z)$ is an entire function, we obtain $\rho(h(z+c)-h(z))<1$. Therefore $C(z)$ must have infinitely many zeros and we arrive at the contradiction.

Subcase 1.2. If $p(z)-q(z)\left[\Delta_{c}^{m-1}(q(z+c)-q(z))\right] \not \equiv 0$, then

$$
\begin{gathered}
{\left[h(z)\left(\Delta_{c}^{m-1}\left(h_{1}(z)-h(z)\right)\right)-C(z)\right] e^{2 \alpha z^{s}}+\left[h(z)\left(\Delta_{c}^{m-1}(q(z+c)-q(z))\right)+q(z)\left(\Delta_{c}^{m-1}\left(h_{1}(z)-h(z)\right)\right)\right] e^{\alpha z^{s}}} \\
=p(z)-q(z)\left[\Delta_{c}^{m-1}(q(z+c)-q(z))\right] .
\end{gathered}
$$

Let

$$
f_{1}(z)=\frac{\left[h(z)\left(\Delta_{c}^{m-1}\left(h_{1}(z)-h(z)\right)\right)-C(z)\right]}{p(z)-q(z)\left[\Delta_{c}^{m-1}(q(z+c)-q(z))\right]} e^{2 \alpha z^{s}}
$$

and

$$
f_{2}(z)=\frac{\left[h(z)\left(\Delta_{c}^{m-1}(q(z+c)-q(z))\right)+q(z)\left(\Delta_{c}^{m-1}\left(h_{1}(z)-h(z)\right)\right)\right]}{p(z)-q(z)\left[\Delta_{c}^{m-1}(q(z+c)-q(z))\right]} e^{\alpha z^{s}}
$$

Thus, $f_{1}(z)+f_{2}(z)=1$. It follows from the second main theorem that

$$
T\left(r, f_{1}\right) \leq N\left(r, f_{1}\right)+N\left(r, \frac{1}{f_{1}}\right)+N\left(r, \frac{1}{f_{1}-1}\right)+S\left(r, f_{1}\right) \leq N\left(r, \frac{1}{f_{1}}\right)+N\left(r, \frac{1}{f_{2}}\right)+S\left(r, f_{1}\right) \leq O\left(r^{s-1+\varepsilon}\right)+S\left(r, f_{1}\right)
$$

which is contradiction with $\rho\left(f_{1}\right)=s$. Thus, $\left[f(z)^{n} \Delta_{c}^{m} f\right]^{(k)}-p(z)$ has infinitely many zeros.

Case 2. If $k \geq 1$, then it follows from (7) and (8) that

$$
\left[\left(q(z)+h(z) e^{\alpha z^{s}}\right)^{n}\left(\Delta_{c}^{m-1}\left(q_{1}(z)+h_{2}(z) e^{\alpha z^{s}}\right)\right)\right]^{(k)}-C(z) e^{\gamma z^{s}}=p(z)
$$

This yields

$$
\begin{aligned}
{\left[q(z)^{n} \Delta_{c}^{m-1}\left(q_{1}(z)\right)\right.} & +\Delta_{c}^{m-1}\left(D_{1}(z) e^{\alpha z^{s}}+\ldots+D_{j}(z) e^{j \alpha z^{s}}+\ldots+D_{n}(z) e^{n \alpha z^{s}}\right. \\
& \left.\left.+h(z) h_{2}(z) e^{(n+1) \alpha z^{s}}\right)\right]^{(k)}-C(z) e^{\gamma z^{s}}=p(z)
\end{aligned}
$$

where

$$
D_{j}(z)=C_{n}^{j} q(z)^{n-j} q(z+c) h(z)^{j}+C_{n}^{j-1} q(z)^{n-j+1} h_{1}(z) h(z)^{j-1}
$$


and $\rho\left(D_{j}(z)\right)<s, j=1, \ldots, n$. For any integer $\mathrm{k}$, from (13), we obtain

$$
F_{1}(z) e^{\alpha z^{s}}+\ldots+F_{j}(z) e^{j \alpha z^{s}}+\ldots+F_{n}(z) e^{n \alpha z^{s}}+F_{n+1}(z) e^{(n+1) \alpha z^{s}}-C(z) e^{\gamma z^{s}}=p(z)-\left[q(z)^{n} \Delta_{c}^{m-1}\left(q_{1}(z)\right)\right]^{(k)}
$$

where $F_{j}(z)$ are differential polynomials of $h(z), h_{1}(z), q(z)$ and $q(z+c)$, and their powers of derivatives and in addition, $\rho\left(F_{j}(z)\right)<s, j=1, \ldots, n+1$. In what follows we consider two cases.

Subcase 2.1. If $p(z)-\left[q(z)^{n} \Delta_{c}^{m-1}\left(q_{1}(z)\right)\right]^{(k)} \equiv 0$ in Lemma 8 , then all $F_{j}(z) \equiv 0, j=1,2, \ldots n$, and $F_{n+1}(z)-C(z) \equiv 0$. We state that $n=1$; otherwise, let $n \geq 2$. If $k=1$ in $F_{1}(z) \equiv 0$, then we get

$$
D_{1}^{\prime}(z)+\alpha s z^{s-1} D_{1}(z)=0
$$

which gives the nontrivial solution $D(z)$ of the first-order differential equation presented above satisfying the equality $\rho(D(z))=s$ in contradiction with the condition $\rho(D(z))<s$. Thus, $D_{1}(z) \equiv 0$.

For $k=2$, let

$$
g(z)=D_{1}^{\prime}(z)+\alpha s z^{s-1} D_{1}(z)=0 .
$$

Thus we have $g^{\prime}(z)+\alpha s z^{s-1} g(z)=0$, which also implies that $\rho(g(z))=s$ in contradiction with the condition $\rho(g(z))=\rho(D(z))<s$. By using this method for any positive integer $k$, we conclude that $D_{1}(z) \equiv 0$, that is,

$$
C_{n}^{1} q(z)^{n-1} q(z+c) h(z)+q(z)^{n} h(z+c) e^{\alpha\left(C_{s}^{1} z^{s-1} c+\ldots+C_{s}^{s-1} z c^{s-1}+c^{s}\right)} \equiv 0 .
$$

From Lemma 9, we get $s=1$, which implies that

$$
C_{n}^{1} q(z)^{n-1} q(z+c) h(z)+q(z)^{n} h(z+c) e^{\alpha c} \equiv 0 .
$$

In view of Lemma 10, since $\rho(h(z))<1$, the degree of $C_{n}^{1} q(z)^{n-1} q(z+c)+q(z)^{n} e^{\alpha c}$ must be smaller than the degree of $q(z)^{n}$. Hence, we arrive at the equality

$$
e^{\alpha c}=-C_{n}^{1}
$$

provided that $q(z)$ is not a constant. By using the same arguments as above, we also get $D_{2}(z) \equiv 0$, that is

$$
C_{n}^{2} q(z)^{n-2} q(z+c) h(z)^{2}+C_{n}^{1} q(z)^{n-1} h(z+c) e^{\alpha c} h(z) \equiv 0 .
$$

As above, if $q(z)$ is not a constant, then we find

$$
C_{n}^{1} e^{\alpha c}=-C_{n}^{2}
$$

It follows from (16) and (17) that $n=0$; a contradiction. Thus, we have $n=1$. By using (13) and Lemmas 8 and 7 , we obtain $D_{1}(z) \equiv 0$. Hence (15) is equivalent to (11). Therefore, we get

$$
f(z)=q(z)+h e^{\alpha z}
$$

where $e^{\alpha c}=1$.

Subcase 2.2. If $p(z)-\left[q(z)^{n} \Delta_{c}^{m-1}\left(q_{1}(z)\right)\right]^{(k)} \not \equiv 0$, then we combine $\rho\left(F_{j}(z)\right)<s$ and $\rho(C(z))<s$ with (14) and Lemma 7 we obtain

$$
\left[F_{n+1}(z)-C(z)\right] e^{(n+1) \alpha z^{s}}=p(z)-\left[q(z)^{n} \Delta_{c}^{m-1}\left(q_{1}(z)\right)\right]^{(k)}
$$

or

$$
F_{j}(z) e^{j \alpha z^{s}}=p(z)-\left[q(z)^{n} \Delta_{c}^{m-1}\left(q_{1}(z)\right)\right]^{(k)}
$$

which is impossible. Thus, $\left[f(z)^{n} \Delta_{c}^{m} f\right]^{(k)}-p(z)$ has infinitely many zeros. 


\section{Acknowledgement}

The authors are grateful to the referees for their remarks. The first author is supported by Ref. No. F.510/3/DRS-III/2016(SAP-I) Dated:29th Feb.2016 and the second author is supported by URS fellowship, Department of Mathematics, Karnatak University, Dharwad, Ref No. KU/Sch/URS/2017-18/468.

\section{Competing interests}

The authors declare that they have no competing interests.

\section{Authors' contributions}

All authors have contributed to all parts of the article. All authors read and approved the final manuscript.

\section{References}

[1] Bergweiler W. and Eremenko A., On the singularities of the inverse to a meromorphic function of finite order, Rev. Mat. Iberoam.,11, 355-373(1995).

[2] Bergweiler W. and Langley J. K., Zeros of difference of meromorphic functions, Math. Proc. Cambridge Philos. Soc., 142,133-147 (2007).

[3] Chen H. H. and Fang M. L., On the value distribution of $f^{n} f^{\prime}$, Sci. China. Ser. A, 38, 789-798 (1995).

[4] Chen Z. X., Huang Z. B., and Zheng X.M., On properties of difference polynomials, Acta Math. Sci. Ser. B Eng. Ed., 31, No. 2,627-633 (2011).

[5] Chen Z. X., Growth and zeros of meromorphic solution of some linear difference equations, J. Math. Anal. Appl., 373, 235241(2011).

[6] Chiang Y. M., Feng S. J., On the Nevanlinna characteristic of $f(z+\eta)$ and difference equations in the complex plane, Ramanujan J.,16:105-129 (2008).

[7] Goldberg A. A. and Ostrovskii I. V., Value Distribution of Meromorphic Functions, Transl. Math. Monographs, Vol. 236, Amer. Math. Soc., Providence, RI (2008).

[8] Halburd R. G. and Korhonen R. J., Difference analogue of the lemma on the logarithmic derivative with application to difference equations, J. Math. Anal. Appl., 314, 477-487 (2006).

[9] Halburd R. G. and Korhonen R. J., Meromorphic solutions of difference equations, integrability, and the discrete PainlevÂ'e equations,J. Phys. A, 40, 1-38 (2007).

[10] Hayman W. K., Picard values of meromorphic functions and their derivatives, Ann. of Math. (2),70, 9-42 (1959).

[11] Hayman W. K., Meromorphic functions, Claredon Press, Oxford, 1964.

[12] Hayman W. K., Slowly growing integral and subharmonic functions, Comment. Math. Helv., 34, 75-84 (1960).

[13] Huang Z. B. and Chen Z. X., A Clunie lemma for difference and q-difference polynomials, Bull. Aust. Math. Soc., 81, 23-32 (2010).

[14] Laine I. and Yang C. C., Value distribution of difference polynomials, Proc. Japan Acad. Ser. A Math. Sci., 83, 148-151 (2007).

[15] Liu K. and Yang L. Z., Value distribution of the difference operator, Arch. Math.(Basel), 92, 270-278 (2009).

[16] Liu K., Liu X. L., and Cao T. B., Some results on zeros and uniqueness of difference-differential polynomials, Appl. Math. J.Chinese Univ. Ser. A, 27, 94-104 (2012).

[17] Liu K., Cao T.B. and Liu X. L., The properties of differential-difference polynomials, Ukrainian Math. J., Vol.69, No.1, June,(2017).

[18] Mohonho A., Nevanlinna characteristics of certain meromorphic functions, Teor. Funkts., Funkts. Anal. Prilozh., 14, 83-87 (1971).

[19] Wang Y. F. and Fang M. L.,Picard values and normal families of meromorphic functions with multiple zeros, Acta Math. Sinica (N.S.), 14, No. 1, 17-26 (1998).

[20] Yang C. C. and Yi H. X., Uniqueness Theory of Meromorphic Functions, Kluwer Academic Publishers, Dordrecht,2003; Chinese original: Science Press, Beijing, 1995.

[21] Yang L., Normality for families of meromorphic functions, Sci. Sinica. Ser. A,29, No.12, 1263-1274 (1986).

[22] Zhang J. L., Value distribution and shared sets of differences of meromorphic functions, J. Math. Anal. Appl., 367, 401-408 (2010). 